\title{
Approcci contemporanei all'etica dei farmacisti e la loro valutazione alla luce dei princìpi della bioetica personalista
}

\author{
Współczesne ujęcia etyki farmaceutów i ich ocena w świetle zasad bioetyki personalistycznej
}

\author{
Wojciech Kućko \\ Faculty of Family Studies, Cardinal Stefan Wyszyński University in Warsaw, Poland \\ ORCID: https://orcid.org/0000-0003-4271-8478•w.kucko@uksw.edu.pl \\ Received: 09 Jul 2020; Revised: 23 Aug 2020; Accepted: 24 Aug 2020
}

Sommario: Lo scopo dell'articolo è quello di mostrare lo stato attuale della ricerca sull'etica dei farmacisti ed i suoi approcci contemporanei alla luce delle varie prospettive bioetiche. II grande sviluppo delle scienze tecniche, biologiche, chimiche e biomediche suscita molte questioni etiche legate alla trasformazione della vita umana e del mondo. Particolarmente impegnativi sono i cambiamenti che riguardano la vita umana dal concepimento alla sua morte naturale, i quali si applicano a tutte le professioni sanitarie, compresa la professione farmaceutica, perché i compiti del farmacista vanno oltre le normali attività commerciali. Nel testo dell'articolo viene presentato lo status quaestionis riguardo all'etica dei farmacisti dopo di che vengono discussi e analizzati vari approcci etici: prospettiva storica, deontologia farmaceutica ed etica delle virtù. Alla luce dell'insufficienza di tali modelli, viene esibita una visione personalistica della professione del farmacista, con il riferimento alla bioetica personalistica. Essa offre un'opportunità adeguata e completa per rispondere ai dilemmi morali relativi all'esercizio di questa professione. Tra le varie questioni, si richiama l'attenzione sul dimostrare il farmacista come custode della vita umana, I'umanizzazione della professione, la questione della responsabilità e l'obiezione di coscienza. L'analisi è condotta alla luce dei principi della bioetica personalistica, con riferimento ai documenti più importanti della Chiesa cattolica. L'approccio personalista sembra affrontare in modo più completo la complessità del lavoro del farmacista, con particolare enfasi sulla questione del suo sviluppo personale in stretta connessione con la cura della salute del paziente.

Parole chiave: bioetica, bioetica personalista, etica, etica dei farmacisti, farmacista

Streszczenie: Celem artykułu jest ukazanie obecnego stanu badań nad etyką farmaceutów oraz współczesnych jej ujęć w świetle różnych systemów bioetycznych. Wielki rozwój nauk technicznych, biologicznych, chemicznych i biomedycznych jest źródłem wielu pytań etycznych, związanych z przemianami życia człowieka i świata. Szczególnym wyzwaniem są zwłaszcza te przemiany, które dotyczą życia człowieka od poczęcia aż do naturalnej śmierci i związane są ze wszystkimi zawodami medycznymi, wśród których znajduje się także profesja farmaceutyczna. Zadania farmaceuty wykraczają bowiem poza zwyczajne działania komercyjne. W tekście artykułu ukazany jest status quaestionis, dotyczący etyki farmaceutów. Następnie zostają omówione i przeanalizowane różne jego ujęcia etyczne: perspektywa historyczna, deontologia farmaceutyczna oraz etyka cnót. W świetle niewystarczalności takich modeli ukazane jest z kolei personalistyczne ujęcie profesji farmaceuty, odwołujące się do bioetyki personalistycznej. Ta ostatnia daje bowiem adekwatną i całościową możliwość odpowiedzi na dylematy moralne, związane z wykonywaniem tego zawodu. Wśród różnych kwestii zwraca się uwagę na ukazanie farmaceuty jako stróża życia człowieka, humanizację zawodu, zagadnienie odpowiedzialności oraz sprzeciwu sumienia. Analiza zostaje przeprowadzona w świetle zasad bioetyki personalistycznej, z odwołaniem do ważniejszych do- 
kumentów Kościoła katolickiego. Ujęcie personalistyczne wydaje się w najpełniejszy sposób podejmować złożoność pracy aptekarza, ze szczególnym uwzględnieniem kwestii jego rozwoju osobowego w ścisłym związku z dbałością o zdrowie pacjenta.

Słowa kluczowe: etyka, etyka farmaceutów, farmaceuta, bioetyka, bioetyka personalistyczna

\section{Introduzione}

Esercitare la professione del farmacista esige non soltanto la capacità intellettuale, la scienza medica o le abilità psico-fisiche. Il farmacista resta sempre nell'ambito del servizio alla vita, il bene primario dell'uomo. Il compito dell'articolo è quello di presentare i modelli contemporanei dell'etica farmaceutica con particolare enfasi sulla bioetica personalistica, nonché di determinare quali sfide devono affrontare i farmacisti nel contesto dell'esercizio della loro professione per il bene della società ${ }^{1}$.

È vero, come ha affermato Paolo VI, che la Chiesa e la teologia non sono le istanze competenti nelle questioni mediche (Paolo VI 1964, 139-140), ma questo non significa che la teologia non possa introdurre qualcosa di significativo nella medicina. Proprio l'uomo è l'oggetto comune della ricerca, sia per la medicina che per la teologia, sempre da diversi punti di vista. Dall'altro lato è vero come ha affermato Galeno, il più famoso medico dell'antichità (II sec., accanto a Ippocrate) - che non si può essere un buon medico senza conoscere la filosofia e senza praticarla (Angeletti e Gazzaniga 2008, 4344). Perciò la teologia, con il suo contributo alla bioetica personalista, si interessa alla medicina, al suo sviluppo e al suo progresso

1 La base di questa presentazione panoramica proviene dalla ricerca per il dottorato, finalizzata con la tesi: Etica dei farmacisti in Italia. Verso una nuova comprensione e prassi alla luce della bioetica personalista, Pontificia Università Lateranense Accademia Alfonsiana, Romae 2016, realizzata con il sostegno della borsa del Comitato Regionale di Coordinametno delle Università del Lazio e Conferenza dei Rettori delle Università Pontificie Romane. tanto quanto si interessa dell'uomo e del suo bene.

\section{Lo status quaestionis dell'etica dei farmacisti}

Il grande sviluppo delle scienze tecniche, biologiche e chimiche, specialmente quelle della biomedicina, suscita interesse e crea una sfida particolare, anche per l'etica, nella quale si può notare l'interessamento crescente della bioetica. Infatti, la riflessione bioetica rappresenta una fondamentale modalità di attenzione alle problematiche della contemporaneità e vi si gioca la possibilità di prospettare un futuro degno di esistenza. In questo campo dell'etica della vita, bisogna sottolineare anche l'importanza del lavoro dei professionisti sanitari, cioè di persone preparate, attraverso studi specialistici, nella difesa e nella cura della vita umana.

Il giusto desiderio dell'umanità di ricercare la salute e il benessere, prevedendo le malattie e impedendone la diffusione, ha dato inizio al progresso non soltanto dell'industria farmaceutica e delle ricerche su nuovi farmaci, ma ha provocato anche tante domande sulla legittimità e moralità del lavoro farmaceutico. La divulgazione dei farmaci, il vasto accesso alla vendita nelle società sviluppate, generano alcuni problemi e dubbi per il paziente, il quale si rivolge al farmacista come la prima persona competente per ricevere un orientamento e un consiglio in caso di malattia o delle angustie per la propria salute. Dall'altra parte, la povertà, in qualche Paese del mondo, i danni e le disuguaglianze nella società e nelle nazioni, provocano anche questioni sulla giustizia distributiva e quella commutativa, per quanto riguarda la realizzazione del diritto dell'uomo alla difesa della propria vita e salute. 
Il farmacista (dal greco $\phi \alpha \rho \mu \alpha ́ \alpha \sigma \omega ~-~ " c u-$ rare con medicinali", "preparare, insaporire”, "essere avvelenato") (Montanari 2005, 2256), come collaboratore naturale del medico, è consapevole della nobiltà e dell'importanza della sua missione. Anche la sua professione, carica di una grande responsabilità, non è più, come in passato, quella di attendere, nel chiuso del suo laboratorio, all'allestimento dei farmaci, ma è diventata quella di essere il primo consulente medico e anche il distributore dei medicinali, il quale, in questa ottica, acquista una forte valenza etica e morale perché impegna la professionalità e la coscienza farmaceutica nella protezione della salute e della vita delle persone che a lui si rivolgono.

Questo compito, inevitabilmente, fa riferimento all'etica, perché ogni sistema medico è fondato sull'antropologia concreta, creando, in tal modo, una concezione etica, una gerarchia di valori, e di conseguenza, un modo di ragionare pratico, dando risposte concrete alle domande. L'incarico del farmacista va oltre la semplice occupazione commerciale, perché richiede una profonda comprensione dei problemi personali del paziente, ma anche gli aspetti etici fondamentali dei servizi resi alla vita e alla dignità della persona umana. Spiegando questo compito, il Papa polacco ha affermato: "Conscia della novità e della complessità dei problemi posti dal progresso della scienza e delle tecniche, la Chiesa fa ascoltare più spesso la sua voce e dà chiare indicazioni al personale della sanità di cui i farmacisti fanno parte" (Giovanni Paolo II 1992, 992).

Già una ricerca generale nelle bibliografie sull'etica medica mostra che il tema dell'etica dei farmacisti, particolarmente nel campo della bioetica cattolica, non è assai approfondito. Si possono indicare alcune opere (in modo particolare inglesi, spagnole e tedesche) che affrontano l'oggetto in chiave utilitarista o liberale (Valverde e Nacle 1999; Buerki e Vottero 2002; Salek and Edgar 2002; Wingfield e Badcott 2007; Fink und Tromm 2015; Veatch and Haddad e Last $\left.2017^{3}\right)$. Inoltre, bisogna notare alcuni libri di deontologia farmaceutica oppure alcune voci nei dizionari e nelle enciclopedie bioetiche recenti che si riferiscono alla prospettiva etica e bioetica (Masino 1934; Binetti 1974; Boccardo 1982; Mallarini 2001; Tripaldi 2004, 487-492; Soldini 2004, 882-888; Cicconetti 2013, 235249; Brusa e Baratta 2013). Tuttavia, manca un'elaborazione complessiva dell'etica dei farmacisti dal punto di vista della morale cattolica, ce ne sono soltanto pochi approcci alla questione (Dréano 1986, 67-69; Tomás y Garrido 1997, 679-588; Tripaldi 1997, 441445; Tettamanzi 2002, 203-209; Villano 2007; Tomás y Garrido 2020). Abbastanza ben sviluppato appare il problema dell'obiezione di coscienza del farmacista, specialmente nell'ambito del biodiritto (Agulles Simó 2006; Wicclair 2006, 225-250; De Rosa 2007, 480-485; Grześkowiak 2008, 202-239; Prusak 2015).

Occorre allora esaminare quali sono i modelli principali dell'etica dei farmacisti. Verranno proposti i cinque approcci che emergono dalla bibliografia dell'oggetto: quello storico, l'impostazione deontologica, alcuni modelli pragmatico-utilitaristi e dell'etica delle virtù nonché i punti essenziali del modello personalista.

\section{L'approccio storico all'etica dei farmacisti}

L'etica medica si immedesima con la storia della sofferenza umana che gli uomini vogliono combattere in diversi modi, grazie alle scoperte della medicina contro il dolore e le malattie. La medicina ellenica è ritenuta una culla della deontologia medica grazie alle sue scuole medico-filosofiche, la Ionica, l'Italica e quelle di Cnido e di Coo (circa 550500 anni a.C.). Qui si già intravede l'umanizzazione della medicina che si avvicinava al malato, considerato essere umano da curare nella sua casa o nei luoghi pubblici, ma comunque fuori dall'ambiente dei templi. Da questo momento non c'è più il medico al centro dell'interesse professionale, bensì il malato e i suoi problemi di cui si dovrebbe occupare il medico. Dall'intero Corpus 
Hippocraticum emerge una visione globale della medicina, secondo la quale qualsiasi terapia non si limita a curare i sintomi, ma deve entrare nella struttura psicofisica del paziente, abbracciando anche il contesto della sua vita. Il Corpus contiene il famoso Giuramento: "un capolavoro di humanitas" valido e attuale fino ai nostri tempi (Spinsanti 1982, 144-159) ed è diventato il punto di riferimento di quasi ogni considerazione dell'etica sanitaria, anche quella dei farmacisti.

Nel panorama biblico-patristico apparisce il tema poco trattato della figura del medicofarmacista, che, in modo particolare, sulle pagine dei libri sapienziali e del Vangelo si riferisce a Dio Creatore (cfr. Sir 38,1-15), a Cristo Pastore, al Buon Samaritano (cfr. $L c$ 10,25-37) e all'uomo (Fasce 2009).

I secoli successivi vennero arricchiti dai medici ebrei, fra cui Asaph ben Berachyahu (VI sec.), il quale, nel suo Giuramento, proibì la somministrazione dei veleni e delle pratiche magiche. Un ebreo più famoso, Mosè Maimònide (1135-1204), allievo di Averroè, scrisse una preghiera celebre (non considerata oggi un giuramento, ma un'invocazione a Dio). Essa contiene indicazioni preziose pure per il servizio farmaceutico, mettendo al primo posto l'umiltà e il rispetto verso Dio e verso il paziente (Puccini 1995, 11). Un importante contributo all'etica medica del Medioevo venne dato dai rappresentanti del mondo arabo fra cui per es. Alì Rabbàn al Tàbari (838-870) (Puccini 1995, 11). Intanto l'etica medica occidentale del Medioevo consiste in due filoni principali: quello della medicina monastica e quello degli insegnamenti della famosa Scuola Salernitana. Un rappresentante di essa, Colofone il Giovane, nel suo trattato De Arte Medendi (c. anno 1120) ammonisce i farmacisti: "Chiunque voglia preparare medicine, per prima cosa deve valutare che le droghe usate siano buone e non siano rovinate perché vecchie... le droghe dovranno essere convenientemente raccolte, essiccate e ben custodite" (Signore 2014, 93). Nella Scuola, la sua l'impronta la lasciò anche san Tommaso d'Aquino. La crescita della consapevolezza circa l'identità e il ruolo dei farmacisti nella società viene consolidata con la fondazione delle Corporazioni o Arti degli Speziali, sin dalla metà del '20o. Fra le norme etiche principali si possono indicare quelle che, secoli prima, aveva già stabilito Ippocrate: l'obbligo del segreto professionale, il divieto di fornire veleni o abortivi e la moderazione nei confronti del guadagno (Puccini 1995, 13).

Con il pontificato di Martino $\mathrm{V}$ e la bolla dell' 8 marzo 1429, la quale istituì l'Universitas Aromatariorum, iniziarono i lavori che affrontavano le questioni dell'arte degli Speziali di Roma, ma anche quelle sull'etica professionale (Signore 2014, 106116). Un esempio notevole della nuova normativa è rappresentato dal Ricettario Fiorentino, redatto dal Collegio dei Medici e Speziali (dalla sua prima edizione del 1498, sino alla sesta, nel 1789), inteso non solo come la prima farmacopea ufficiale, ma anche come una raccolta delle più importanti indicazioni etiche (Signore 2014, 164-170).

Le riflessioni deontologiche si possono trovare in opere particolari, chiamate Galatei, cioè trattati stampati e proliferati in Europa, soprattutto nell'Ottocento, rivolti ai giovani medici e farmacisti, con lo scopo di portare un miglioramento dei comportamenti e delle abitudini nel contesto sanitario (Baldini e Malavasi 2008, 12-23).

Il volume Medical Ethics del medico di Manchester Thomas Percival (1740-1804), edito per la prima volta nel 1803 , indicò un nuovo percorso nella storia dell'etica medica. Percival dedica la terza parte della sua opera ai rapporti tra medico e farmacista $(O f$ the Conduct of Physicians Towards Apothecaries), in cui esige la cooperazione amichevole in favore del paziente. Il ruolo del farmacista dovrebbe essere quello di aiutare il paziente, ma anche quello di saper agire nei confronti dei problemi di salute, osservati nei sintomi esterni del paziente (Percival 1803). Nell'ultimo secolo del Secondo Millennio, i principi deontologici dei medici e dei farmacisti, acquistarono una nuova 
valenza perché, all'orizzonte del dibattito si profilò la Giurisprudenza medica, con le sue leggi civili, penali e amministrative, con relativi regolamenti e codici di carattere sanitario (Puccini 1995, 18-23). Dopo la Prima guerra mondiale, oltre al ragionamento deontologico, non mancavano le pubblicazioni d'ispirazione cristiana, in cui la professione medica e quella farmaceutica rivendicavano il carattere della vocazione. Fra gli autori italiani si ricorda Cristoforo Masino (1907-1988), per il quale le parole chiave per esprimere il ruolo del farmacista erano: responsabilità, cooperazione e morale della persona del farmacista (Masino 1934). Dopo le esperienze terribili della Seconda Guerra mondiale sono stati elaborati, presso le diverse organizzazioni, documenti importanti riguardanti la morale medica, profanata e abusata da alcuni medici nazisti e comunisti, per es. il Codice internazionale di etica medica, promulgato nel 1949. Negli ultimi decenni nacquero diversi approcci moderni all'etica dei farmacisti, che vengono presentati in seguito.

\section{I lineamenti principali della deontologia farmaceutica}

Il termine deontologia deriva da due vocaboli grechi: $\delta \varepsilon \dot{\varepsilon} \omega v$, -оvтоৎ (dovere,

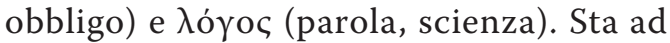
indicare la scienza di ciò che si ha il dovere di fare per poter raggiungere un determinato scopo dell'atto. Il primo a utilizzarlo $\mathrm{fu}$ Jeremiah Bentham (1748-1832), il quale, nell'epoca dell'illuminismo e dell'utilitarismo inglese, nella sua opera Deontology, or the Science of Morality, intendeva esporre la deontologia come disciplina delle azioni umane non sottoposte a qualche legge positiva (Bentham 1834). Non è difficile osservare che essa offre una morale al minimo, intesa come scienza dell'opportuno, dove si calcolano le conseguenze delle azioni, optando per quella che comporta non solo il benessere delle persone, ma prende anche in considerazione altri valori. In tal modo, la deontologia viene opposta alle diverse forme del consequenzialismo morale, secondo cui solo le conseguenze dell'agire indicano il valore morale delle norme, delle leggi o degli atti stessi dell'uomo. Bentham sottolineava anche che l'unico principio, sul quale si possono basare le società e le leggi, sarebbe il vantaggio che ne deriva per tutti, vale a dire il principio dell'utile comune (Silvestri 2011, 151-152). In Italia, i principii deontologici dei farmacisti vengono precisati nel Codice deontologico del Farmacista, il quale è in vigore dal 2007 (Federazione Nazionale degli Ordini dei Farmacisti Italiani 2012). Per es. in Gran Bretagna, un'organizzazione di antica tradizione, che si occupa della professione farmaceutica e promuove le diverse iniziative riguardanti la professione, è la Royal Pharmaceutical Society of Great Britain (RPSGB), fondata nel 1841.

Sulla base dei testi degli autori che sostengono l'approccio deontologico e prendendo in esame le norme generali della deontologia medica par excellence, si può provare a costruire l'impalcatura della deontologia farmaceutica, indicando le sue idee principali: (1) La varietà delle norme deontologiche. Già la lettura generale dei manuali e dei Codici farmaceutici offre una prima considerazione: si possono distinguere diverse categorie di norme deontologiche. (2) «Primum non nocere». Tra i vari principi, questo antico detto, attribuito a Ippocrate, resta il più importante. Il servizio del farmacista, come quello del medico non può mettere a rischio il valore della vita ed arrecare un danno al paziente. (3) Il rapporto con il paziente. Il nucleo dell'interesse deontologico è quello di costruire buoni rapporti tra professionista e paziente. (4) La correttezza. Essa si esprime, prima di tutto, nella competenza professionale, ma anche nell'osservanza delle regole del Codice. Secondo il ragionamento deontologico, la fedeltà alle norme garantisce un buon esercizio e il raggiungimento degli scopi prefissati. (5) I rapporti professionali. Il farmacista non agisce a vuoto, ma le proprie decisioni e tutta la sua attività si svolgono nell'ambito sanitario. Dalla distinzione delle professioni (quella medica e quella farmaceutica), emerge la separazione delle 
competenze: il farmacista non deve fare il medico. (6) Il segreto professionale. Questo viene considerato come una delle basi del servizio onesto del farmacista (Rizzo 1968, 501-502; Spinsanti 1993, 31-33; Herranz 2011, 155-168).

Dal punto di vista dello sviluppo del pensiero bioetico (in modo particolare di quello del modello personalista e pure dall'odierna conoscenza dell'antropologia umana) la proposta della deontologia appare insufficiente. Le norme deontologiche non sono interiorizzate dall'operatore sanitario, ma rappresentano una morale eteronoma, seconda la quale il compimento dell'atto buono garantisce i buoni risultati. La deontologia farmaceutica non possiede un fondamento filosofico solido che possa proteggere più la persona che la norma di legge. Nei confronti con il diritto medico, essa viene considerata poco precisa: priva di fondamento della filosofia del diritto e di un regime disciplinare. Infine, la deontologia farmaceutica sembra racchiudere nelle sue norme i privilegi dell' élite, indipendentemente dagli interessi della società (Herranz 2011, 158).

\section{II modello pragmatico-utilitarista}

Tra i modelli più diffusi della bioetica e della farmacia c'è quello dei principi. Esso è stato ampliato dagli scienziati Tom L. Beauchamp (1939-) e James F. Childress (1940-) a causa della necessità della riflessione teorico-pratica, che è nata nel contesto del grande progresso scientifico e teologico nell'ambiente medico e biomedico. La fonte del paradigma è la ragione umana, sulla cui base si possono distinguere quattro princìpi che potrebbero essere un aiuto utile per poter affrontare le questioni bioetiche nella pratica di farmacisti. La proposta si riferisce alle teorie etiche (ethical teories) che concorrono a definire e pianificare le regole (principles and rules) che farebbero da guida al comportamento e al giudizio pratico di un operatore sanitario (Beauchamp and Childress 1994). In questo senso, la teoria dei princìi sarebbe un'etica normativa, applicata alla pratica medica.
Riguardo la struttura dei princìpi, gli autori sopra citati ne distinguono quattro categorie: (1) il rispetto dell'autonomia (respect for autonomy), (2) la non maleficenza (nonmaleficence), (3) la beneficenza (beneficence), (4) la giustizia (justice) (Beauchamp e Childress 1994). In questa prospettiva, si prende a questo punto in considerazione l'opera Prospettive e deontologia della professione farmaceutica di Francesco Binetti, edita nel 1974, ai tempi della grande riforma sanitaria in Italia. Egli suggerisce i seguenti princìpi per i farmacisti: la professione stessa, intesa come un impegno per la difesa e la promozione della vita umana (art. 32 della Costituzione Italiana); la responsabilità professionale che viene espressa nei confronti del paziente, dello Stato, dell'ambiente sanitario e del farmacista stesso; il segreto professionale; l'importanza della preparazione didattico-sanitaria, terapeutico-psicologica e commerciale; le norme di buona fabbricazione che farebbero parte del principio di "non maleficenza" (Binetti 1974, 11-96).

Il principialismo ha indubbiamente gettato uno sguardo nuovo alla bioetica. In quest'ottica, più adatta a risolvere i casi concreti, si può valutare il bene dell'atto nella diversità delle conseguenze a delle alternative dell'agire. Per questo, viene apprezzata la facoltà razionale del soggetto, la conoscenza dei princìpi che precede la loro applicazione concreta. Alcuni, invece, obiettano a tale paradigma l'inadeguatezza per l'esperienza morale: lo schematismo dei princìi sarebbe eccessivamente astratto. Così, l'applicazione delle norme, nei casi concreti, diventa più importante della considerazione dell'esperienza stessa. Il ragionamento in campo farmaceutico sarebbe focalizzato sull'azione e anche sui casi, ma meno sulla persona del farmacista stesso, come autore dell'agire.

A differenza del paradigma delle virtù che indica il bene e il male dell'atto, il principialismo valuta lo stesso secondo le categorie giusto/sbagliato; l'atto è buono se conforme alla norma. Ma anche qui bisogna formulare il dubbio: occorre stabilire quale sia il bene del paziente, ma anche il bene 
del farmacista a cui lui si rivolge. Per farlo, occorre avere il sostegno della filosofia e di un'adeguata visione dell'uomo. Resta, poi, da valutare la gerarchia dei princìpi stessi, in modo particolare quello della beneficenza e dell'autonomia. Spesso il bene terapeutico del paziente contrasta con la sua autonomia. Valorizzando alcune linee utili, in genere bisogna valutare il paradigma dei princìpi come insufficiente per la pratica farmaceutica.

\section{L'etica delle virtù}

Il paradigma basato sulle virtù fa riferimento alla tradizione filosofica greca della ảpetท́, ad Aristotele, a Tommaso d'Aquino e anche ad altri autori moderni (E.D. Pellegrino, H.T. Engelhardt). Lo stesso viene opposto alle categorie deontologiche neo-kantiane e al modello utilitarista. Si presta maggiore attenzione all'esperienza e alla personalità morale di colui che agisce più che all'atto o alle norme. Nel caso del farmacista che vuole curare un infermo, il problema principale è il fine specifico intrinseco nell'atto. "Le virtù sono dunque quei «tratti del carattere»e della personalità del professionista, ciò che rende il professionista un buon professionista" (Russo 2009). La proposta americana della Ethics of virtue si è divisa in due linee: quella tomistico-aristotelica e quella del relativismo morale.

Edmund D. Pellegrino (1920-2013) ritiene che in questo concetto le virtù, oltre ad essere un elemento essenziale della vita morale, spingono la persona che agisce alle opere virtuose. Le qualità morali che devono accompagnare le abilità tecniche sarebbero: la benevolenza, la fedeltà alla fiducia, la compassione, l'empatia, l'onestà intellettuale, la competenza e la prudenza (Aramini 2009, 29-32). Un altro approccio è proposto da H.T. Engelhardt (1941-) il quale si riferisce solo alla virtù della tolleranza, intesa come rispetto radicale dell'autonomia delle altre persone, perché in essa consiste l'essenza della società secolarizzata e pluralistica. Quel soggettivismo autonomo provoca diversi dilemmi etici, per es. la collaborazione del professionista con il paziente (Aramini 2009, 32-33).

Nell'ottica di questa proposta (VirtueBased Ethics), la più sviluppata nel pensiero nordamericano, lo scopo del farmacista sarebbe quello di diventare una persona buona e acquisire alcune caratteristiche chiamate virtù. L'opera di Robert A. Buerki e Louis D. Vottero rappresenta un tentativo di metterle in pratica. Se un uomo virtuoso commettesse uno sbaglio nel giudizio o un atto morale sbagliato, egli sarebbe meno degno di biasimo di colui che, abitualmente, commettesse lo stesso atto (Buerki and Vottero 2002). Il farmacista guidato dalla virtù della giustizia, distribuirà tutti i rimedi a coloro che ne hanno bisogno, ma osservando le giuste regole della vendita e le circostanze particolari della vita dei pazienti. Un altro farmacista, pieno di fermezza, può rifiutare il commercio di un farmaco abortivo, ma rimane comprensivo e disponibile per coloro che si rivolgessero a lui (Buerki and Vottero 2002, 34-37). Quali virtù dovrebbero costituire la pratica farmaceutica? I suddetti scienziati Buerki e Vottero suggeriscono le seguenti: onestà, dedizione, attenzione e comando di affidabilità. Le tre virtù universali sarebbero invece: altruismo, uguaglianza e giustizia.

Il sistema delle virtù applicato all'etica dei farmacisti appare ambiguo per il suo approccio all'agire del farmacista. Ciò che sembra positivo nella proposta sulle virtù riguardo all'etica farmacisti, è l'impegno continuo nella prassi, nell'esercizio e nel lavoro. Dall'esame della storia di questa teoria risulta la sua connessione al relativismo: il ritorno alla teoria delle virtù nell'etica contemporanea non si basa sul recupero della tradizione aristotelicotomista. L'elenco delle virtù praticabili nella professione medica, fra cui anche quella farmaceutica, secondo tale punto di vista non è fissa, ma dipenderebbe da luogo e tempo, dalla tradizione filosofica e dalle risposte alla domanda sul bene. In tal modo, l'etica non acquista valore normativo, ma diventa una scienza sociologica che 
descrive i diversi comportamenti e valori in un momento specifico della storia. Il nucleo del problema è il riferimento di questa teoria al concetto del bene e della natura umana. Vivere il bene, significa conoscere il significato del bene in quanto persona. Per questo, nella teoria delle virtù appare problematico il suo riferimento all'etica e all'antropologia: se esistono diversi sistemi filosofici, allora, anche le virtù assumono significati diversi, a seconda di essi. Il limite dell'etica delle virtù nel campo della farmacia consiste nell'accentuare il soggetto e, meno, le sue scelte morali. La vita professionale nella ricerca delle virtù può provocare tendenze egoistiche, per cui il farmacista potrebbe concentrarsi più sullo sviluppo della propria personalità che sul bene del paziente (Palazzani 1992, 76-78).

\section{I punti essenziali del modello "personalista"}

Nato in Francia negli anni 30. del XX sec. con Emmanuel Mounier (1905-1950), il personalismo filosofico si concentra sulla persona, concepita come "incarnata in un corpo ed intesa nella sua inoggettivabilità, inviolabilità, libertà, creatività e responsabilità" (Carrasco De Paula 2004, 1359). La via del personalismo, ispirata alla tradizione del pensiero cristiano, è stata riconosciuta come proposta efficace per l'uscita dalla crisi moderna. L'applicazione del personalismo alla bioetica ha sviluppato un grande movimento scientifico, teologico e filosofico per riconsiderare ed affrontare le questioni bioetiche di oggi, alla luce dei valori e della legge naturale. "Per Bioetica personalista si intende una riflessione che affronta le questioni etiche riguardanti la vita umana da una prospettiva che riconosce l'essere e la dignità della persona come valori assoluti, e, di conseguenza, pone come primum principium il rispetto incondizionato della loro inviolabilità e la tutela della loro libera espressione, in primis sul versante dei diritti umani. All'interno della prospettiva personalista il bonum, cioè il valore ultimo che misura l'agire morale, è inteso come promozione dell'essere e della preziosità o dignità della persona in quanto persona" (Carrasco De Paula 2004, 1359). Allora, la proposta della bioetica personalista consiste nella giustificazione dell'etica personalista, fondata sul valore della persona, alla luce del quale si può discernere tra ciò che è tecnicamente possibile e ciò che è eticamente lecito.

Occorre giustificare per quali motivi il modello personalista sembra più adatto nei confronti dei problemi etici dei farmacisti. Si nota che tutti gli altri modelli si definiscono al soggetto, ma ignorano la persona. Elio Sgreccia nota la differenza tra i due termini: “Soggetto è chi, avendo un'essenza, esercita l'esistenza e l'azione; persona vuol dire un certo grado di perfezione ontologica, che indica spiritualità, conoscenza e libertà; un microcosmo che agisce assegnando dei fini a se stessa" (Sgreccia 2007, 218).

Il fondamento della proposta personalista ontologicamente fondata è il concetto della persona, che si riferisce alla verità naturale (scoperta e sviluppata grazie alla ragione umana) e ella verità presente nella morale cattolica (basata sulla Rivelazione divina), esaminata grazie alla Sacra Scrittura e alla Tradizione del Magistero della Chiesa cattolica. Seguendo il pensiero di Elio Sgreccia vengono identificati i seguenti princìi della bioetica personalista che aiutano a concentrarsi sul valore della persona umana (cf. Sgreccia 2007, 221-229).

Il primo valore è la difesa della vita umana. Se il corpo umano ha un valore importante, allora la vita corporea e fisica viene riconosciuta come una qualità fondamentale che non può essere decisa comunque (principio dell'indisponibilità), ed è un valore fondamentale ma non assoluto, perché nella vita fisica non si esaurisce tutta la ricchezza della persona; lo spirito trascende il corpo e il tempo. Ciò che prevale nella vita fisica, è il bene spirituale e assoluto della persona, per il quale è possibile sacrificare la vita corporea. La persona umana è un valore in sé (Sgreccia 2007, 221-224).

Il secondo principio viene chiamato terapeutico oppure della totalità. Il corpo 
umano, pur essendo considerato un tutt'uno, nel caso particolare viene trattato nella terapia (da ciò la denominazione del principio) che dovrebbe rispettare questa condizione, perché spesso, per salvare il tutto o la vita stessa del soggetto, bisogna intervenire sull'organismo o sulle sue parti (Sgreccia 2007, 225-227).

Il terzo principio si riferisce alla libertà e responsabilità. Se il diritto alla difesa della vita precede il diritto alla libertà, ciò significa che la persona deve essere responsabile per la sua vita e per quella degli altri. La libertà non viene qui intesa come assenza di vincoli, ma come una scelta, un modo di autodeterminazione, fondato sui valori, fra i quali il primo è quello della vita (Sgreccia 2007, 224-225).

Il quinto principio è quello di socialità. "Il principio di socialità - osserva E. Sgreccia impegna ogni singola persona a realizzare se stessa nella partecipazione alla realizzazione del bene dei propri simili" (Sgreccia 2007, 227). Salvaguardando la vita e la salute del singolo, si costruisce il bene comune, ma anche la società è responsabile per questi valori delle singole persone. Ciò viene visto, in modo particolare, nei periodi delle malattie contagiose e delle epidemie, in cui la collaborazione di tutti i singoli soggetti e di tutto il mondo medico perseguono il fine del recupero la salute.

L'ultimo principio della bioetica personalista viene collegato con la sussidiarietà, una delle regole fondamentali dell'insegnamento sociale della Chiesa dalla pubblicazione dell'enciclica Quadragesimo anno di Pio XI (1931). La comunità è obbligata all'aiuto di quelle persone che non sono in grado di provvedere alla propria vita e alla propria salute, ma questa ingerenza deve avere i suoi limiti, affinché sia garantita e non venga meno la libera iniziativa dei singoli e dei gruppi (Sgreccia 2007, 228).

\section{Le sfide della professione farmaceutica alla luce della bioetica personalista}

Ora si può chiedere: in che modo il modello personalista può essere collegato con la professione del farmacista?

In primo luogo, si deve affermare che il farmacista dovrebbe essere riconosciuto come ministro della vita. La missione essenziale del farmacista nella sanità è dunque quella "di garantire la qualità e l'efficacia dei farmaci nonché la sicurezza dei pazienti, e in particolare di fare attenzione che il medicamento sia impiegato unicamente per il servizio della vita e il miglioramento dell'individuo sofferente" (Dréano 1986, 67). Il prolungamento della vita e l'ottimizzazione delle risorse pubbliche condizionano il nuovo profilo della farmacia, che è diventata un ponte tra il mondo del benessere (wellness) e quello della medicina classica. La Nuova Carta degli Operatori Sanitari, già nell'introduzione, sottolinea lo scopo del loro lavoro: «L'attività degli operatori sanitari è fondamentalmente un servizio alla vita e alla salute, beni primari della persona umana. A questo servizio dedicano l'attività professionale o volontaria quanti sono impegnati in vario modo nella prevenzione, nella terapia e nella riabilitazione: medici, farmacisti [...]. «La loro professione li vuole custodi e servitori della vita umana»" (Pontificio Consiglio per gli Operatori Sanitari 2016, no. 1).

In secondo luogo, la missione del farmacista fa parte di un processo ben noto nel mondo odierno e considerato l'umanizzazione della medicina, attraverso la quale si intende il cambiamento nella logica della cura: al centro dell'interesse medico c'è la persona del paziente, con tutta la sua dignità e l'integrità psico-fisico-spirituale. Diverse discussioni intorno ai medicamenti contraccettivi (pillola del "giorno dopo", "kit" dell'eutanasia e molte altre) confermano giustamente che al centro del dibattito c'è sempre la vita. Il primo piano operativo potrebbe essere attuato nel diverso rapporto tra farmacista e paziente. Questa relazione appare significativa, perché il farmacista 
spesso è il primo a consigliare, aiutare e parlare con chi è malato. Si percepisce subito l'importanza delle buone maniere, della disponibilità, dell'attenzione posta al paziente, del tempo a lui concesso e della conoscenza dell'operato, che sembrano più importanti dalla professionale o dalla dimensione economica dei rimedi dispensati. In questo senso, la persona del farmacista diventa il farmaco, un sollievo spirituale, talvolta più importante di quello fisico, professionale (Kowalski 2001, 175-195; Leone 2004, 1218).

In terzo luogo, il farmacista è chiamato a sviluppare la propria responsabilità. Secondo Frank Leavitt, la differenza tra la bioetica religiosa e quella laica consiste nell'atteggiamento nei confronti della responsabilità: "Mentre la bioetica secolare ritiene l'autonomia il primo dei principi, la seconda riconosce i diritti alla libertà e all'autonomia come derivati $d a$ e subordinati alla nostra responsabilità" (Leavitt 2004, 1015). Allora, è possibile trattare della responsabilità legale e di coscienza. La responsabilità legale è inseparabilmente collegata alla farmacia. Le leggi, gli editti, i regolamenti, le bolle papali e molti altri documenti regolavano il comportamento del farmacista, ma la responsabilità del farmacista aveva preso piede ancora in anticipo, prima delle leggi che la vollero stabilire. Questa responsabilità si esprime molto concretamente, ma è anche basata sulla fiducia del paziente il quale affidandosi nelle mani del farmacista, espone le condizioni della sua salute (Mazzucco 1949, 5).

Un altro aspetto della responsabilità, oltre a quello nei confronti della legge che obbliga all'esterno, è quello della sua coscienza. In quest'ottica, bisogna sottolineare il compito di ogni persona che deve rendersi se stessa responsabile. È un processo dinamico che si attua nella vita attraverso singoli atti e decisioni morali (la lingua italiana permette di usare il verbo proprio per questo fatto: responsabilizzare, anche nella forma riflessiva responsabilizzarsi) (Sabatini e Colletti 2003, 2216).
Il farmacista si trova ad un crocevia non facile da affrontare: da una parte il medico e il paziente, dall'altra le industrie farmaceutiche, i grossisti, e soprattutto i colleghi. Così "catenato", egli deve avere e usare una coscienza salda e ben formata, nonostante le soluzioni legislative (Casavola 2013, 19-24). Osserva Faggioni: «L'appello alla propria coscienza, anche in assenza di una esplicita previsione di legge, resta un diritto inalienabile del cittadino purché radicato sui valori civili fondamentali e non rechi danno ingiusto ad altri» (Faggioni 2013, 27). In tale prospettiva nel servizio del farmacista si può distinguere: il diritto all'obiezione di coscienza (nel caso della contraccezione) e il dovere dell'obiezione (nei confronti dei prodotti abortivi). L'operato del farmacista non è un semplice mestiere, ma una vocazione che si esprime nella disponibilità al servizio per la vita, partecipando alla costruzione della civiltà della vita. Anche se nei diversi paesi, per es. in Italia e in Polonia, mancano ancora le soluzioni legislative riguardo all'obiezione di coscienza dei farmacisti, essi possono rifiutarsi di compiere alcune pratiche contrarie alla morale cristiana. Il sollevamento dell'obiezione di coscienza rende possibile realizzare pienamente la libertà della persona e la difesa dei valori fondamentali sui quali viene fondata la medicina umanizzata (Comitato Nazionale per la Bioetica 2004; Bramstedt 2006, 1219-1220; Del Bò 2009; Kobyliński 2015). Tale prospettiva viene confermata nel magistero della Chiesa cattolica; Benedetto XVI osserva: "[I farmacisti] hanno un ruolo educativo verso i pazienti per un uso corretto dell'assunzione dei farmaci e soprattutto per far conoscere le implicazioni etiche dell'utilizzazione di alcuni farmaci. In questo ambito, non è possibile anestetizzare le coscienze" (Benedetto XVI 2008, 520). Da queste considerazioni fondamentali emerge chiaramente che il tema dell'obiezione di coscienza richiedere ancora più complessi approfondimenti, dal punto di vista legislativo, etico e medico. 


\section{Conclusioni}

Il compito dell'articolo è stato quello di esaminare gli approcci contemporanei all'etica dei farmacisti e le sfide di questa professione alla luce dei princìi della bioetica personalista. Dalle informazioni presentate emerge chiaramente che l'impostazione etica non è una cosa nuova nel campo farmaceutico, perché sia un esame di storia della farmacia, sia i contributi di filosofia e bioetica, contengono alcune considerazioni morali. Quello che è stato considerato svela chiaramente insufficienza di alcuni sistemi etici per le sfide contemporanee dell'etica dei farmacisti. Il modello personalista e i suoi princìi etici permettono di vedere tutta la persona del farmacista nel contesto del suo complesso lavoro. Da questo emerge apertamente che il farmacista non dovrebbe essere visto come un semplice venditore dei farmaci ma piuttosto come un uomo di coscienza al servizio per la vita umana. Soltanto la prospettiva personalista permette di scrutare adeguatamente le questioni della difesa della vita umana con la sua integrità psico-fisico-spirituale, il ruolo del farmacista nell'umanizzazione della medicina nonché la complessa e attuale domanda sull'obiezione di coscienza nel lavoro farmaceutico. Le affermazioni presentate nell'articolo aprono le tracce per la ricerca delle nuove questioni sull'operato farmaceutico.

\section{Bibliografia}

Agulles Simó, Pau. 2006. La objeción de conciencia farmacéutica en España. Roma: Edizioni Università della Santa Croce.

Angeletti, Luciana R., e Valentina Gazzaniga. $2008^{3}$. Storia, filosofia ed etica generale della medicina. Milano: Elsevier Masson.

Aramini, Michele. $2009^{3}$. Introduzione alla Bioetica . Milano: Giuffrè Editore.

Baldini, Massimo, e Antonello Malavasi (edd.). 2008.

Galatei del medico e del paziente. Roma: Viviani Editore.

Beauchamp, Tom L., and James F. Childress. 1994". Principles of Biomedical Ethics. New York: Oxford University Press.
Benedetto XVI. 2008. "Ai partecipanti al XXV Congresso Internazionale dei Farmacisti Cattolici (29 ottobre 2007)." In Insegnamenti di Benedetto XVI, vol. III/2, 520-522. Città del Vaticano: Libreria Editrice Vaticana.

Bentham, Jeremiah. 1834. Deontology, or the Science of Morality. London-Edinburgh: John Bowring.

Binetti, Francesco. 1974. Prospettive e deontologia professionale farmaceutica. Ausiliare del Corso di Tecnica e Legislazione Farmaceutica. Milano: Casa Editrice Ambrosiana.

Boccardo, Paolo. 1982 ${ }^{2}$. Manuale del farmacista. Milano: Organizzazione Editoriale Medico-Farmaceutica.

Bramstedt, Katrina A. 2006. "When Pharmacists Refuse to Dispense Prescription." The Lancet 367, no. 9518: 1219-1220.

Brusa, Paola, e Francesca Baratta. 2015 . Manuale di legislazione farmaceutica, Torino: Edizioni Libreria Cortina.

Buerki, Robert A., and Louis D. Vottero. 2002². Ethical Responsibility in Pharmacy Practice. Madison: American Institute of the History of Pharmacy.

Carrasco de Paula, Ignacio. 2004. "Personalismo e bioetica." In Enciclopedia di Bioetica e Sessuologia, a cura di Giovanni Russo, 1358-1361. Torino: Ed. Elledici.

Casavola, Francesco P. 2013. "Lectio magistralis: L'obiezione di coscienza tra libertà e responsabilità." I Quaderni di Scienza \& Vita 12: 19-24.

Cicconeti, Andrea. 2013. "Farmacia." In Enciclopedia di Bioetica e Scienza Giuridica, vol. VI: Fallacia naturalistica - Guerra, a cura di Elio Sgreccia e Antonio Tarantino, 235-249. Napoli-Roma: Edizioni Scientifiche Italiane.

Comitato Nazionale per la Bioetica. 2004. Risposta. Nota sulla contraccezione d'emergenza (28 maggio 2004). Accesso: 10.05.2020. http://bioetica. governo.it/media/1868/p59_2004_risposta_ contraccezione_emergenza_it.pdf.

De Rosa, Giuseppe. 2007. "L'obiezione di coscienza dei farmacisti." La Civiltà Cattolica 3779: 480-485.

Del Bò, Corrado. 2009. "Obiezione di coscienza e pillola del giorno dopo.” Bioetica 3: 489-520.

Dréano, Jean. 1986. "Il farmacista davanti alla Bioetica." Dolentium Hominum 2: 67-69. 
Faggioni, Maurizio. 2013. "Non disobbedienza alla legge ma attestazione di coerenza." I Quaderni di Scienza \& Vita 12: 25-28.

Fasce, Silvana. 2009. La lode del medico nel libro biblico del Siracide. Genova: ECIG.

Federazione Nazionale degli Ordini dei Farmacisti Italiani. 2012. "Codice deontologico del Farmacista. Testo approvato dal Consiglio Nazionale il 19.6.2007." In Codice di deontologia forense e delle altre professioni: 2012, a cura di Remo Danovi, 255-265. Milano: Giuffrè.

Fink, Erika, und Cornelia Tromm. 2015. Pharmazie und Ethik. Vom Umgang mit Menschen in der Apotheke 2015. Eschborn: Govi Verlag.

Giovanni Paolo II. 1992. "Discorso alla Federazione Internazionale dei Farmacisti Cattolici (3 novembre 1990)." In Insegnamenti di Giovanni Paolo II, vol. XIII/2, 990-993. Città del Vaticano: Libreria Editrice Vaticana.

Grześkowiak, Alicja. 2008. "Obiezione di coscienza per categorie professionali particolari (farmacisti, giudici, amministrativi, consulenti etc.)." In $\mathrm{La}$ coscienza cristiana a sostegno del diritto alla vita. Atti della Tredicesima Assemblea della Pontificia Accademia per la Vita (Città del Vaticano, 2325 febbraio 2007), a cura di Elio Sgreccia e Jean Laffitte, 202-239. Città del Vaticano: Libreria Editrice Vaticana.

Herranz, Gonzal. 2011. "Deontologia. Parte etica." In Enciclopedia di Bioetica e Scienza Giuridica vol. IV: Danno alla salute - Duplice effetto, a cura di Elio Sgreccia e Antonio Tarantino, 155165. Napoli-Roma: Edizioni Scientifiche Italiane. Kobyliński, Andrzej. 2015. "Etyczne i prawne aspekty stosowania preparatu medycznego ellaOne czyli tzw. pigułki «pięć dni później»." Studia Ecologiae et Bioethicae 13(1): 9-34.

Kowalski, Edmund. 2001. "Quale uomo e quale etica per la bioetica. Una proposta di umanizzazione della riflessione bioetica." Studia Moralia 39(1): 175-195.

Leavitt, Frank. 2004. "Responsabilità medica." In Nuovo Dizionario di Bioetica, a cura di Salvino Leone, e Salvatore Privitera, 1015-1018. RomaAcireale: Città Nuova - Istituto Siciliano di Bioetica.

Leone, Salvino. 2004. "Umanizzazione." In Nuovo Dizionario di Bioetica, a cura di Salvino Leone, e Salvatore Privitera, 1216-1220. Roma-Acireale: Città Nuova - Istituto Siciliano di Bioetica.

Mallarini, Erika. 2001. Farmacista: il futuro della professione. Ruoli, attività e competenze. Milano: Franco Angeli.

Masino, Cristoforo. 1934. Quesiti di moralità professionale del farmacista. Responsabilità della cooperazione. Roma: Editrice Studium.

Mazzucco, Romolo. "Responsabilità del farmacista di fronte all'individuo e alla collettività." Raphäel 2(1-2): 5 .

Montanari, Franco (ed.). $2005^{2}$. Vocabolario della lingua greca. Torino: Loescher Editore.

Palazzani, Laura. 1992. "Bioetica dei principi e bioetica delle virtù: il dibattito attuale negli Stati Uniti." Medicina e Morale 1: 59-85.

Paolo VI. 1964. "Discours aux participants au Congrès International de Chirurgie et au Congrès de la Société de Chirurgie Cardiovasculaire (20 settembre 1963)." In Insegnamenti di Paolo VI, vol. I, 139-140. Città del Vaticano: Libreria Editrice Vaticana.

Percival, Thomas. 1803. Medical Ethics or a Code of Institutes and Precepts Adapted to the Professional Conduct of Physicians and Surgeons. Manchester: Printed by S. Russell.

Pontificio Consiglio per gli Operatori Sanitari (per la Pastorale della Salute). 2016. Nuova Carta degli Operatori Sanitari. Città del Vaticano: Libreria Editrice Vaticana.

Prusak, Małgorzata. 2015. Sprzeciw sumienia farmaceutów. Aspekty etyczne, teologiczne i prawne. Kraków: Wydawnictwo św. Stanisława BM.

Puccini, Clemente. 1995. "Profilo storico dell'etica medica e della deontologia professionale." Rivista di Storia della Medicina 5(2): 7-23.

Rizzo, Carlo. 1968. "Deontologia farmaceutica." In Dizionario di Teologia Morale, a cura di Francesco Roberti e Pietro Palazzini, 501-503. Roma $1968^{4}$ : Editrice Studium.

Russo, Giovanni. 2009. Bioetica medica. Per medici e professionisti della sanità. Messina-Torino: Cooperativa San Tommaso - Elledici.

Sabatini, Francesco, e Vittorio Coletti (ed.). 2003. Dizionario della Lingua Italiana. Milano: Rizzoli Larousse S.p.A. 
Salek, Sam, and Andrew Edgar (eds.). 2002. Pharmaceutical Ethics. Chichester: John Wiley \& Sons, LTD.

Sgreccia, Elio. $2007^{4}$. Manuale di bioetica, vol. I: Fondamenti ed etica biomedica. Milano: Vita e Pensiero.

Signore, Giancarlo. 2014. Storia della Farmacia. Dalle origini al XXI secolo. Milano: Edra.

Silvestri, Nicola. 2011. "Deontologia. Parte medica." In Enciclopedia di Bioetica e Scienza Giuridica vol. IV: Danno alla salute - Duplice effetto, a cura di Elio Sgreccia e Antonio Tarantino, 151-155. NapoliRoma: Edizioni Scientifiche Italiane.

Soldini, Maurizio. 2004. "Farmacista." In Enciclopedia di Bioetica e Sessuologia, a cura di Giovanni Russo, 882-888. Torino: Ed. Elledici.

Spinsanti, Sandro. 1982. "L'ethos ippocratico." Medicina e Morale 2: 144-159.

Spinsanti, Sandro. 1993. Bioetica in sanità. RomaFirenze: NIS - Giunta Regionale Toscana.

Tettamanzi, Dionigi. 2002. "Farmacista." In Dizionario di Bioetica, a cura di Marco Doldi, 203-209. Casale Monferrato: Piemme.

Tomás y Garrido, Gloria M. 1997. "Bioética farmacéutica ¿Avance o retroceso?" Cuadernos de Bioética no. $1^{\mathrm{a}}$ : 679-688.

Tomás y Garrido, Gloria M. 2020. "Bioética farmacéutica." Accesso: 10.06.2020. https:// enciclopediadebioetica.com/mod/page/view. php?id=3295.

Tripaldi, Elia. 1997. "Farmacista." In Dizionario di Teologia Pastorale Sanitaria, a cura di Efisio Locci e Carlo Rocchetta e Luciano Sandrin, 441-445. Torino: Edizioni Camilliane.

Tripaldi, Elia. 2004. "Farmacista." In Nuovo Dizionario di Bioetica, a cura di Salvino Leone e Salvatore Privitera, 487-492. Roma-Acireale: Città Nuova - Istituto Siciliano di Bioetica.

Valverde, José L., e Pilar Arrebola Nacle (edd.). 1999. Estudios de ética farmacéutica. Madrid: Ediciones Doce Calles S.L.

Veatch, Robert M., Amy Haddad, and E. J. Last. $2017^{3}$. Case Studies in Pharmacy Ethics. New York: Oxford University Press.

Villano, Raimondo. 2007. La cruna dell'ago: meridiani farmaceutici tra etica laica e morale cristiana. Milano: Chiron Foundation.

Wicclair, Mark R. 2006. "Pharmacies, Pharmacists and Conscientious Objection." Kennedy Institute of Ethics Journal 16(3): 225-250.

Wingfield, Joy, and David Badcott. 2007. Pharmacy Ethics and Decision Making. London-Chicago: Pharmaceutical Press. 